Pacific Journal of Mathematics

ON THE FUNCTIONAL REPRESENTATION OF CERTAIN 


\title{
ON THE FUNCTIONAL REPRESENTATION OF CERTAIN ALGEBRAIC SYSTEMS
}

\author{
J. H. Williamson
}

1. Introduction, Definitions and Examples. In this paper an attempt is made to generalize the well-known representation theory of commutative Banach algebras by functions on the maximal ideals of the algebra [4]. The present paper is devoted almost exclusively to algebraic questions; topological aspects of the theory will be treated elsewhere.

In considering commutative algebras $A$ over the complex field $C$, there are relatively few cases in which one can assert that the quotient $A / M$ of the algebra by a maximal ideal is isomorphic to $C$. Apart from Banach algebras, there are the locally $m$-convex algebras of E.A. Michael [6] and R. Arens [1], and the 'algèbres à inverse continu' of L. Waelbroeck [8], [9] (=Q-algebras, in the terminology of Kaplansky [5], with continuous inversion). There are many interesting algebras which do not belong to either of these classes, and it would be desirable to have a theory to cover them as far as possible.

The basic idea is derived from the classical work of Carleman, von Neumann, and Stone on unbounded self-adjoint linear operators $T$ in Hilbert space (see, for example, [7]). Here the analysis is carried out with the aid of the bounded transformations $(T-\lambda I)^{-1}$; the spectrum of $T$ is the set of complex numbers $\lambda$ such that $(T-\lambda I)^{-1}$ does not exist as a bounded transformation. This suggests that if we start with a commutative algebra $A$, and a suitable sub-algebra $B$ (corresponding to the 'bounded' elements of $A$ ) we may be able to effect a useful analysis of $A$, and somehow represent an element $a \in A$ by a function whose values are those complex numbers $\lambda$ such that $(a-\lambda e)^{-1}$ does not exists in $B$ ( $e$ being the unit of $A$ ). It turns out that this is basically correct, although there are certain complications of detail. For instance, the representing functions may take infinite values; this is unavoidable. The space on which the functions are defined is that of the 'maximal $B$-ideals' or ' maximal ordinary $B$-ideals' of the algebra, not the space of maximal ideals in the ordinary sense.

Much of the theory of this paper applies to algebras over fields of fairly general type; for instance, many results are true for any algebraically closed field. It is no more difficult to develop the theory for the general case than for the case of the complex field. Let $K$ be any

Received December 24, 1955. This paper was written while the author was a Commonwealth Fund Fellow. 
(commutative) field, $A$ a commutative linear algebra over $K$, with a unit $e$, and $B$ a sub-algebra of $A$, containing $e$. A restriction will presently be put on $B$ (immediately following Lemma 4), and after Theorem 1 , $K$ will be taken to be algebraically closed. Further special assumptions on $A$ and $K$ will be made in the later sections of the paper.

\section{Definition 1. A subset $J$ of $A$ is a $B$-ideal of $A$ if}

(i) $x-y \in J$ whenever $x \in J, y \in J$, and

(ii) $x b \in J$ whenever $x \in J, b \in B$.

The $B$-ideal $J$ is admissible if $e \notin J$; it is ordinary if $x y \in J$ whenever $x \in J, y \in J$; otherwise it is exceptional. $(B$-ideal $=B$-submodule; ordinary $B$-ideal $=B$-submodule which is a sub-algebra).

It may be useful to remark that a $B$-ideal which is a proper subset of $A$ is not necessarily an admissible $B$-ideal, by the above definition. For instance, $B$ itself is clearly a $B$-ideal of $A$; it may be a proper subset of $A$ but it is never an admissible $B$-ideal.

We give now one or two examples of the type of system under consideration.

(i) Let $A$ be any algebra of the type specified above, and take $B=A$. The $B$-ideals of $A$ are the ideals (in the usual sense) of $A$; all are ordinary.

(ii) Let $A$ be as in (i), and take $B=K e$ (which we shall sometimes write as $K$, if no danger of confusion exists). The $B$-ideals of $A$ are the linear subspaces of $A$.

In particular, let $A$ be the algebra of pairs of complex numbers $\left(a_{1}, a_{2}\right)$, with pointwise addition and multiplication. The admissible $B$ ideals of $A$ are the proper linear subspaces not containing $(1,1)$. They are thus (a) the element $(0,0)$, and (b) for each complex $\alpha \neq 1$, the subspace generated by $(1, \alpha)$, and the subspace generated by $(0,1)$. There are precisely three ordinary admissible $B$-ideals, namely $(0,0)$ and those generated by $(0,1)$ and $(1,0)$.

(iii) Let $A$ be the algebra of polynomials, with complex coefficients, in the indeterminate $t$, and let $B$ be the sub-algebra of constants. The sets $\left\{a: a\left(t_{0}\right)=0\right\} \quad\left(t_{0}\right.$ a complex number) are clearly ordinary $B$-ideals of $A$. An elementary argument shows that they are maximal admissible ordinary $B$-ideals; it will appear later (after Theorem 2) that these are the only such $B$-ideals.

(iv) As for (iii), but with 'polynomial' replaced by ' rational function'. Here the maximal ordinary $B$-ideals are the sets $\left\{a: a\left(t_{0}\right)=0\right\}$ for each complex $t_{0}$ and the set $\{a: a(\infty)=0\}$.

(v) Let $A$ be the algebra of (equivalence-classes of) complex almost everywhere finite Lebesgue measurable functions on $(0,1), B$ the sub- 
algebra of essentially bounded functions. Among the $B$-ideals of $A$ are (a) the set of all functions of $A$ which are zero (almost everywhere) on $E$, for any fixed subset $E$ of $(0,1)$ of positive measure (this is an ordinary $B$-ideal, and in fact an ideal); and (b) the set of functions $f(t)$ such that $|f(t)| \leqq k n^{-1}$ almost everywhere in $E_{n}$, for each $n$, where $E_{n}$ is a decreasing sequence of measurable sets such that the measure of $E_{n}$ tends to zero as $n$ tends to infinity ( $k$ depends on $f$ only). This is an ordinary $B$-ideal, but not an ideal.

(vi) Let $A$ be an algebra of (possibly unbounded) self-adjoint or normal linear transformations of a Hilbert space into itself, and let $B$ be the sub-algebra of bounded operators. This type of algebra will be considered in $\$ 7$.

In what follows it will be important to distinguish clearly between ordinary maximal $B$-ideals, that is, admissible $B$-ideals which are ordinary and which are not properly contained in any admissible $B$-ideal, and maximal ordinary $B$-ideals, that is, admissible $B$-ideals which are ordinary and which are not properly contained in any admissible ordinary $B$-ideal (maximal $=$ maximal admissible). In example (ii) above, all the $B$-ideals (b) are clearly maximal. Of these only the ideals generated by $(0,1)$ and $(1,0)$ are ordinary; and these two are clearly also the only maximal ordinary $B$-ideals of $A$.

Lemma 1. (i) If $J$ is a maximal B-ideal of $A$, then $B \cap J$ is a maximal ideal of $B$; if $I$ is a maximal ideal of $B$, there is a maximal $B$-ideal of $A$ containing $I$.

(ii) If $J$ is a maximal ordinary $B$-ideal of $A$, then $B \cap J$ is a maximal ideal of $B$; if $I$ is a maximal ideal of $B$, there is a maximal ordinary $B$-ideal of $A$ containing $I$.

Proof. (i) It is clear that $B \cap J$ is a proper ideal of $B$. Suppose that $J^{\prime}$ is a proper ideal of $B$ which properly contains $B \cap J$; then $J$ $+J^{\prime}$ is a $B$-ideal of $A$ which properly contains $J$ and does not contain $e$. Since $J$ was assumed to be maximal, this is a contradiction, and so $B \cap J$ is a maximal ideal of $B$.

The second assertion follows, by a simple application of Zorn's lemma, from the fact that any proper ideal of $B$ is an admissible $B$ ideal of $A$, and the fact that the union of an ascending chain of admissible $B$-ideals is clearly an admissible $B$-ideal.

(ii) As for (i), with ' $B$-ideal' replaced by 'ordinary $B$-ideal'.

In general, a maximal ideal of $B$ is contained in many maximal (or maximal ordinary) $B$-ideals of $A$; but in some cases it is possible to assert that the extension is unique; see $\S 4$, Proposition 5 and $\S 7$, 
Lemma 13.

2. A representation theorem. Let $\infty$ be a symbol such that $\infty+\lambda$ $=\infty$ for all $\lambda \in K, \infty \cdot \infty=\infty$ and $\lambda \infty=\infty$ for all nonzero $\lambda \in K$. Denote the field $K$ augmented by $\infty$ by the symbol $K^{\prime}$. Now let $J$ be any linear subspace of $A$, not containing $e$. Define a function with values in $K^{\prime}$ as follows:

DEFINITION 2.

$$
f_{J}(a)\left\{\begin{array}{l}
=\lambda \text { if } a-\lambda e \in J, \\
=\infty \text { if } a-\lambda e \notin J \text { for all } \lambda \in K .
\end{array}\right.
$$

It is clear that the function is uniquely defined for all $a \in A$. There are one or two immediate consequences of the definition:

Lemma 2. (i) $f_{J}(\alpha a)=\alpha f_{J}(a)$ for all $\alpha \in K, a \in A(0 \cdot \infty=0$ here $)$.

(ii) $f_{J}\left(a_{1}+a_{2}\right)=f_{J}\left(a_{1}\right)+f_{J}\left(a_{2}\right)$, (when the right-hand side is defined).

Proof. (i) If $f_{J}(a)=\lambda \in K$ then $a-\lambda e \in J$, whence $\alpha a-\alpha \lambda e \in J$ and $f_{J}(\alpha a)=\alpha \lambda=\alpha f_{J}(a)$. If $f_{J}(a)=\infty$, then $a-\lambda e \notin J$ for all $\lambda \in K$; clearly if $\alpha \neq 0$ then $\alpha a-\mu e \notin J$ for all $\mu \in K$, and so $f_{J}(\alpha a)=\infty$. If $\alpha=0$ then $f_{J}(\alpha a)=f_{J}(0)=0$ for all $a \in A$.

(ii) If $f_{J}\left(a_{1}\right)=\lambda_{1} \in K, f_{J}\left(a_{2}\right)=\lambda_{2} \in K$, then $a_{1}+a_{2}-\left(\lambda_{1}+\lambda_{2}\right) e \in K$ and the result follows. If $f_{J}\left(a_{1}\right)=\lambda \in K$, and $f_{J}\left(a_{2}\right)=\infty$, then if $f_{J}\left(a_{1}+a_{2}\right)=\mu \in K$, we would have $f_{J}\left(a_{2}\right)=f_{J}\left(a_{1}+a_{2}-a_{1}\right)=\mu-\lambda \in K$, a contradiction.

Next we turn to the multiplicative properties of the function $f_{J}(a)$. It is clear that if we are to obtain any general results we must take $J$ to be a $B$-ideal of $A$, and moreover an ordinary $B$-ideal; if $J$ is not ordinary we could find $a_{1} \in J, a_{2} \in J$, with $a_{1} a_{2} \notin J$, that is,

$$
f_{J}\left(a_{1} a_{2}\right) \neq f_{J}\left(a_{1}\right) f_{J}\left(a_{2}\right)=0 \text {. }
$$

The first result, however, is valid for any sub-algebra $J$ :

Lemma 3. Let $J$ be any sub-algebra of $A$ not containing $e$. Then if neither of $f_{J}\left(a_{1}\right), f_{J}\left(a_{2}\right)$ is $\infty$, we have $f_{J}\left(a_{1} a_{2}\right)=f_{J}\left(a_{1}\right) f_{J}\left(a_{2}\right)$.

Proof. Let $a_{1}=f_{J}\left(a_{1}\right) e+j_{1}, a_{2}=f_{J}\left(a_{2}\right) e+j_{2}$, where $j_{1} \in J, j_{2} \in J$. Then $a_{1} a_{2}=f_{J}\left(a_{1}\right) f_{J}\left(a_{2}\right) e+f_{J}\left(a_{2}\right) j_{1}+f_{J}\left(a_{1}\right) j_{2}+j_{1} j_{2}$; the required result follows at once.

Difficulties arise when one or both of $f_{J}\left(a_{1}\right), f_{J}\left(a_{2}\right)$ is $\infty$. 
Lemma 4. If $J$ is a sub-algebra of $A$ and $f_{J}(a) \neq \infty$ then $a J \subset J$.

Proof. If $a=\lambda e+j(j \in J)$ then for any $j^{\prime} \in J$ we have $a j^{\prime}=\lambda j^{\prime}$ $+j j^{\prime} \in J$.

For the next lemma, and for all future developments, we require to make the following assumption.

Assumption. If $M$ is any maximal ideal of $B$ then $B / M \cong K$.

This assumption is satisfied in the cases in which we are interested.

Lemma 5. If $J$ is a maximal $B$-ideal of $A$ and $a J \subset J$ then $f_{J}(a)$ $\neq \infty$.

Proof. The result is trivial if $a \in J$; we then have $f_{J}(a)=0$. If $a \notin J$ then $J+a B$ is a $B$-ideal properly containing $J$; since $J$ was maximal, $e=j+a b$ for some $j \in J, b \in B$. We have $b=\lambda e+j^{\prime}$ for some $\lambda \in K$, $j^{\prime} \in J$, by assumption; hence $e-\lambda a=j+a j^{\prime} \in J$. We clearly cannot have $\lambda=0$; hence $a-\lambda^{-1} e \in J$ and $f_{J}(a)=\lambda^{-1} \neq \infty$.

COROLlaRy. If $J$ is maximal and $f_{J}(a)=\infty$, then $e=a j+j^{\prime}$, where $j, j^{\prime} \in J$.

Proof. $a J+J$ is a $B$-ideal of $A$ properly containing $J$ and hence containing $e$.

Lemma 6. If $J$ is an ordinary maximal $B$-ideal of $A$, then $f_{J}\left(a_{1} a_{2}\right)$ $=f_{J}\left(a_{1}\right) f_{J}\left(a_{2}\right)$, whenever the right-hand side is defined.

Proof. The case in which $f_{J}\left(a_{1}\right)$ and $f_{J}\left(a_{2}\right)$ are both finite has already been covered (Lemma 3). Suppose then that $f_{J}\left(a_{1}\right)=\infty$. By Lemma 5, Corollary, we have $e=a_{1} j+j^{\prime}$, where $j, j^{\prime} \in J$. If $a_{2}-\lambda e=j^{\prime \prime} \in J(\lambda \neq 0)$ we have $a_{1} a_{2} j=\lambda e+j^{\prime \prime}-\lambda j^{\prime}-j^{\prime} j^{\prime \prime} \notin J$, whence $f_{J}\left(a_{1} a_{2}\right)=\infty$, by Lemma 4 . If $e=a_{2} j_{1}+j_{2}\left(j_{1}, j_{2} \in J\right)$ then $a_{1} a_{2} j_{j}=e-j^{\prime}-j_{2}+j^{\prime} j_{2} \notin J$, whence $f_{J}\left(a_{1} a_{2}\right)$ $=\infty$ as before.

We can now collect the results obtained.

Theorem 1. Let $\mathscr{F}_{0}$ be the set of ordinary maximal $B$-ideals of $A$. Then there is a mapping of $A$ into the set of $K^{\prime}$-valued functions on $\mathscr{J}_{0}: a \rightarrow f_{J}(a)$, so that the structure of $A$ is preserved as far as it can be, that is $f_{J}(\alpha a)=\alpha f_{J}(a), f_{J}\left(a_{1}+a_{2}\right)=f_{J}\left(a_{1}\right)+f_{J}\left(a_{2}\right)$, and $f_{J}\left(a_{1} a_{2}\right)=f_{J}\left(a_{1}\right) f_{J}\left(a_{2}\right)$, whenever the right-hand sides of these equalities are defined. 
The above theorem has one serious flaw; given $A$ and $B$, the set

may be empty. For example, let $A$ be a field properly containing $K$, and take $B=K e$. Then any maximal $B$-ideal is a maximal linear subspace of $A$ not containing $e$; any $a \in A$ can be expressed uniquely as $a$ $=\lambda e+j$, where $j \in J$. If $J$ were ordinary we would have $a J \subset J+J J$ $=J$, that is, $J$ would be an ideal of $A$ in the usual sense, which is impossible.

It is uncertain whether, given $A$, it is possible to choose $B$ so that there is at least one ordinary maximal $B$-ideal. In any case, $B$ will often be prescribed in advance, so that no choice is possible.

We are thus obliged to look at maximal ordinary $B$-ideals rather than ordinary maximal $B$-ideals. We have, by Lemma 1 (ii), the assurance that there always exist at least as many maximal ordinary $B$ ideals of $A$ as there are maximal ideals of $B$, that is, always at least one.

3. A better representation theorem. We now consider maximal ordinary $B$-ideals instead of maximal $B$-ideals. This introduces some technical difficulties (which can, however, be overcome), and also makes it necessary to confine attention to fields $K$ which are algebraically closed. We shall make this assumption from now on. The sort of difficulty which arises if the field is not algebraically closed is adequately illustrated by considering the complex field $C$, as an algebra over the real field $R$. Here there is a unique maximal ordinary $R$-ideal $J$ $=\{0\}$; if $a$ is any complex number with a nonzero imaginary part, then $f_{J}(a)=\infty$. Clearly the multiplicative properties of $f$ are quite unsatisfactory.

Lemma 7. If $J$ is a maximal ordinary $B$-ideal and $a J \subset J$ then $f_{J}(a) \neq \infty$.

Proof. If $a \in J$ then $f_{J}(a)=0$; suppose then that $a \notin J$. The set $J+a B+a^{2} B+\cdots$ is an ordinary $B$-ideal of $A$, properly containing $J$; hence $e=j+a b_{1}+a^{2} b_{2}+\cdots+a^{n} b_{n}$ for some $j \in J, b_{1}, \cdots, b_{n} \in B$. We shall show that we can take $n=1$ here. First, it is to be noted that there is no loss of generality in supposing that $b_{1}, \cdots, b_{n}$ are all scalar multiples of $e$; if in the above representation we had $b_{r}=\lambda_{r} e+j_{r}(1 \leqq r \leqq n)$ then we could also write

$$
e=j^{\prime}+\lambda_{1} a+\lambda_{2} a^{2}+\cdots+\lambda_{n} a^{n} \text {, where } j^{\prime}=j+j_{1} a+\cdots+j_{n} a^{n} \in J .
$$

Second, it is clearly permissible to assume that the representation of $e$ in this way is of minimum degree. We do this. Let $\mu(a)$ be a polynomial in $a$, with coefficients in $K$, which is in $J$, and of minimum 
degree. Assume that the degree of $\mu$ is $n>1$. Let $(\alpha-\alpha e)$ be a factor of $\mu(a)$, and write $\mu(a)=(a-\alpha e) x$. By assumption $x \notin J$, and so the set $J+x B+x^{2} B+\cdots$ is an ordinary $B$-ideal of $A$, properly containing $J$. We thus have

$$
e=j^{\prime \prime}+x b_{1}^{\prime}+\cdots+x^{m} b_{m}^{\prime}, \text { where } j^{\prime \prime} \in J, b_{1}^{\prime}, \cdots, b_{m}^{\prime} \in B .
$$

This gives

$$
a-\alpha e=(a-\alpha e) j^{\prime \prime}+\mu(a) b_{1}^{\prime}+x \mu(a) b_{2}^{\prime}+\cdots+x^{m-1} \mu(a) b_{m}^{\prime} \in J,
$$

which contradicts the assumption that $\mu(a)$ was of minimum degree. Thus $\mu(a)=a-\alpha e$, and $f_{J}(a)=\alpha \neq \infty$.

COROLlary. If $J$ is a maximal ordinary $B$-ideal and $f_{J}(a)=\infty$ then

$$
e=j_{0}+a j_{1}+a^{2} j_{2}+\cdots+a^{n} j_{n} \text { for some } j, j_{1}, \cdots, j_{n} \in J \text {. }
$$

Proof. The set $J+a J+a^{2} J+\cdots$ is an ordinary $B$-ideal of $A$, properly containing $J$. Hence it contains $e$.

It will appear later (Lemma 9, Corollary) that we can always take $n=1$ in this representation. In the meantime it is convenient to formulate this as follows.

Property $P$. Let $J$ be a maximal ordinary $B$-ideal of $A$, and $a$ an element of $A$ such that $f_{J}(a)=\infty$. We shall say that property $P$ holds (for $a$ and $J$ ) if $e=a j+j^{\prime}$ for some $j, j^{\prime} \in J$.

LEMMA 8. If $f_{J}(a)=\infty$, and property $P$ does not hold, then we can find $j^{*} \in J$ such that $f_{J}\left(a j^{*}\right)=\infty$.

Proof. Clearly, by Lemma 7 we can find $j^{*}$ such that $a j^{*} \notin J$. If $f_{J}\left(a j^{*}\right)=\alpha$, we would have $a j^{*}-\alpha e \in J$, whence $e=\alpha^{-1} a j^{*}+j^{\prime}$, that is, property $P$ would hold. Since we assume the contrary, $f_{J}\left(a j^{*}\right)=\infty$.

Lemma 9. If $J$ is a maximal ordinary $B$-ideal of $A$, then $f_{J}\left(a_{1} a_{2}\right)$ $=f_{J}\left(a_{1}\right) f_{J}\left(a_{2}\right)$, whenever the right-hand side is defined.

Proof. (a) The case in which both factors on the right are finite has already been covered (Lemma 3).

(b) Suppose that $f_{J}\left(a_{1}\right)=\infty$, with property $P$, and $f_{J}\left(a_{2}\right)=\alpha(\neq 0)$. Then $e=a_{1} j+j^{\prime}, a_{2}=\alpha e+j^{\prime \prime}$, so that

$$
a_{1} a_{2} j=\alpha e-\alpha j^{\prime}+j^{\prime \prime}-j^{\prime} j^{\prime \prime} \notin J .
$$


Hence $f_{J}\left(a_{1} a_{2}\right)=\infty$, by Lemma 4 .

(c) Suppose $f_{J}\left(a_{1}\right)=f_{J}\left(a_{2}\right)=\infty$, property $P$ holding for both. Then $e=a_{1} j_{1}+j_{1}^{\prime}, e=a_{2} j_{2}+j_{2}^{\prime}$, and so

$$
a_{1} a_{2} j_{1} j_{2}=e-j_{1}^{\prime}-j_{2}^{\prime}+j_{1}^{\prime} j_{2}^{\prime} \notin J,
$$

whence $f_{J}\left(a_{i} a_{2}\right)=\infty$, as before.

(d) Now suppose that $f_{J}\left(a_{1}\right)=\infty$, property $P$ not being true, and $f_{J}\left(a_{2}\right)=\alpha \neq 0$. We shall show that $f_{J}\left(a_{1} a_{2}\right)=\beta \in K$ is impossible. Let

$$
e=j_{0}+j_{1} a_{1}+\cdots+j_{n} a_{1}^{n},
$$

and $a_{1} a_{2}=\beta e+j$. Then

$$
a_{2}^{n}=j_{0} a_{2}^{n}+j_{1}(\beta e+j) a_{2}^{n-1}+\cdots+j_{n}(\beta e+j)^{n},
$$

that is,

$$
\left(a e+j^{\prime}\right)^{n}=j_{0}\left(\alpha e+j^{\prime}\right)^{n}+j_{1}(\beta e+j)\left(\alpha e+j^{\prime}\right)^{n-1}+\cdots+j_{n}(\beta e+j)^{n} .
$$

This gives at once $\alpha^{n} e \in J$, which is impossible. Hence $f_{J}\left(a_{1} a_{2}\right)=\infty$.

(e) Finally suppose that $f_{J}\left(a_{1}\right)=\infty$, property $P$ not holding, and $f_{J}\left(a_{2}\right)=\infty$ (property $P$ possibly holding, possibly not). We note first that it will be enough to prove that under these hypotheses $f_{J}\left(a_{1} a_{2}\right)=0$ is impossible. For, if $f_{J}\left(a_{1} a_{2}\right)=\alpha \neq 0$, we could choose $j^{*}$ as in Lemma 8 , and replace $a_{1}$ by $a_{1} j^{*}$. Then property $P$ fails to hold for $a_{1} j^{*}$, and we would have $f_{J}\left(a_{1} j^{*}\right)=f_{J}\left(a_{2}\right)=\infty, f_{J}\left(a_{1} j^{*} a_{2}\right)=0$. So, assume that $f_{J}\left(a_{1} a_{2}\right)$ $=0$. Let

$$
e=j_{0}+j_{1} a_{1}+\cdots+j_{m} a_{1}^{m}=j_{0}^{\prime}+j_{1}^{\prime} a_{2}+\cdots+j_{n}^{\prime} a_{2}^{n},
$$

where $m$ and $n$ are minimal. It is clearly no restriction to assume that $m \geqq n$. If $a_{1} a_{2}=j \in J$, then

$$
a_{1}^{n}\left(e-j_{0}^{\prime}\right)=j_{1}^{\prime} j a_{1}^{n-1}+\cdots+j_{n}^{\prime} j^{n} .
$$

Multiply this by $j_{m} a_{1}^{m-n}$, and we have

$$
j_{m} a_{1}^{m}\left(e-j_{0}^{\prime}\right)=j_{m} j_{1}^{\prime} j a_{1}^{m-1}+\cdots+j_{m} j_{n}^{\prime} j^{n} a_{1}^{m-n} .
$$

But also

$$
j_{m} a_{1}^{m}\left(e-j_{0}^{\prime}\right)=\left(e-j_{0}-j_{1} a_{1}-\cdots-j_{m-1} a_{1}^{m-1}\right)\left(e-j_{0}^{\prime}\right),
$$

so that, equating the right-hand sides of the last two equations, we have an expression for $e$ as a polynomial in $a_{1}$, with coefficients in $J$, and of degree $\leqq m-1$, which contradicts the assumed minimality of $m$. Thus $f_{J}\left(a_{1} a_{2}\right)=\infty$ in this case also.

The above five cases exhaust all the possibilities, and so the lemma is proved. 
Corollary. Property $P$ always holds; that is, if $J$ is a maximal ordinary $B$-ideal and $f_{J}(a)=\infty$, then $e=a j+j^{\prime}$ for some $j, j^{\prime} \in J$.

Proof. By Lemma 7, Corollary, we have $e=a h+j^{\prime}$, where $h=j_{1}$ $+\cdots+j_{n} a^{n-1}$. By Lemma 9, we must have $f_{J}(h)=0$, that is, $h \in J$.

As in the case of maximal $B$-ideals, we collect our results:

THEOREM 2. Let $\mathscr{J}_{0}^{\prime}$ be the set of maximal ordinary B-ideals of $A$. Then there is a mapping of $A$ into the set of $K^{\prime}$-valued functions on $\mathscr{J}_{0}^{\prime}: a \rightarrow f_{J}(a)$, so that $f_{J}(\alpha a)=\alpha f_{J}(a), f_{J}\left(a_{1}+a_{2}\right)=f_{J}\left(a_{1}\right)+f_{J}\left(a_{2}\right)$ and $f_{J}\left(a_{1} a_{2}\right)=f_{J}\left(a_{1}\right) f_{J}\left(a_{2}\right)$, whenever the right-hand sides of these equalities are defined.

Since, as has been remarked, there always exists a maximal ordinary $B$-ideal of $A$, Theorem 2 always has content.

We can now show, as promised, that the $B$-ideals specified in Example (iii) of $\S 1$ are the only maximal ordinary $B$-ideals. Suppose that there is a maximal ordinary $B$-ideal $J$ such that $f_{J}(t)=\infty$. Then, by Lemma $9, f_{J}(a)=\infty$ for every non-constant polynomial $a \in A$; that is, $J=\{0\}$, which is clearly not maximal. Thus $f_{J}(t)$ is always finite, from which it follows at once that $J$ is one of the specified $B$-ideals.

It may be noted that if $J$ is a maximal ordinary (or ordinary maximal) $B$-ideal of $A$, then the function $f_{J}(a)$ has the properties

(1) $f_{J}(e)=1 ; f_{J}(b) \in K$ for all $b \in B$, and

(2) $f_{J}(\alpha a)=\alpha f_{J}(a), f_{J}\left(a a^{\prime}\right)=f_{J}(a) f_{J}\left(a^{\prime}\right), f_{J}\left(a+a^{\prime}\right)=f_{J}(a)+f_{J}\left(a^{\prime}\right)$ whenever the right-hand sides are defined. Conversely, if we have a function $f$ with these properties, the set $\{a: f(a)=0\}$ is clearly an ordinary $B$-ideal of $A$ but not in general a maximal one (consider Example (iii) of $\S 1$ and write $f(a)=\alpha$ if $a=\alpha$ (constant), $f(a)=\infty$ otherwise). This is in contrast to the situation in which $J$ is an ideal and $f$ a genuine homomorphism.

4. Further general results. The spectrum, etc. We shall for the most part be concerned with maximal ordinary $B$-ideals; in one or two cases we consider maximal $B$-ideals (which may or may not be ordinary).

Definition 3. Denote by $B_{2}$ the set of elements of $A$ such that $f_{J}(a)$ is finite for all maximal ordinary $B$-ideals $J$, and by $B_{2}^{\prime}$ the set such that $f_{J}(a)$ is finite for all maximal $B$-ideals $J$. If $B=B_{2}$, we say that $B$ is strongly saturated; if $B=B_{2}^{\prime}$, then $B$ is said to be weakly saturated.

It is evident that $B_{2} \supseteqq B_{2}^{\prime} \supseteqq B$. 
Proposition 1. (i) $B_{2}$ is a sub-algebra of $A$.

(ii) The maximal ordinary $B_{2}$-ideals of $A$ are the same as the maximal ordinary $B$-ideals of $A$.

(iii) If $M$ is any maximal ideal of $B_{2}$, then $B_{2} / M \cong K$.

(iv) $\left(B_{2}\right)_{2}=B_{2}$, for any $B$.

Proof. (i) This is an immediate consequence of Lemma 3. (Note that in general $B_{2}^{\prime}$ is not a sub-algebra of $A$ ).

(ii) Clearly every $B_{2}$-ideal of $A$ is also a $B$-ideal, since $B_{2} \supseteqq B$. On the other hand, by Lemma 3 every ordinary $B$-ideal is also an ordinary $B_{2}$-ideal. Hence the result follows.

(iii) By Lemma 1 (ii) the maximal ideals of $B_{2}$ are the traces on $B_{2}$ of the maximal ordinary $B_{2}$-ideals of $A$, that is, of the maximal ordinary $B$-ideals. Hence, for any $M$ and any $a \in B_{2}$, we have $a-\alpha e$ $\in M$ for some $\alpha \in K$, that is, $B_{2} / M \cong K$.

(iv) This follows at once from (ii).

The last part of the above proposition shows that for any $A$ it is always possible to choose a strongly saturated sub-algebra $B ;(K e)_{2}$ is of the required type.

Theorem 3. (i) The element $a \in A$ has an inverse $a^{-1} \in B$ if and only if it is in no maximal $B$-ideal of $A$.

(ii) The element $a \in A$ has an inverse $a^{-1} \in B_{2}$ if and only if it is in no maximal ordinary $B$-ideal of $A$. If such an inverse exists, it is expressible as a polynomial in a with coefficients in $B$.

Proof. (i) If $a a^{-1}=e$, where $a^{-1} \in B$, then clearly a cannot be in any admissible $B$-ideal of $A$. If $a b \neq e$ for all $b \in B$, then $a B$ is an admissible $B$-ideal of $A$, and hence is contained in some maximal $B$-ideal $J$. Then $a=a e \in J$.

(ii) If $J$ is a maximal ordinary $B$-ideal, it is also a maximal ordinary $B_{2}$-ideal, by Proposition 1 (ii). Thus if $a \in J$, the relation $e=a a^{-1}$, with $a^{-1} \in B_{2}$, is impossible.

If $a$ is such that $e$ is not expressible as a polynomial in $a$, with coefficients in $B$ and without constant term, then the set of all such polynomials clearly forms an admissible ordinary $B$-ideal of $A$. There is thus a maximal ordinary $B$-ideal containing $a$. So, if $a \notin J$ for all maximal ordinary $J$, it follows that $e=a a^{-1}$, where $a^{-1}$ is expressed as a polynomial in $a$ with coefficients in $B$. By Lemma 9 , since $f_{J}(a)$ is never zero it follows that $f_{J}\left(a^{-1}\right)$ is never infinite, that is, $a^{-1} \in B_{2}$.

CoRollary. If $B$ is strongly saturated, the element $a \in A$ has an 
inverse in $B$ if and only if it is in no maximal ordinary $B$-ideal.

In general the expression for $a^{-1}$ as a polynomial in $a$ will necessarily be of degree $\geqq 1$. Consider, for example, the algebra of $\S 1$, Example (ii). If $\alpha \neq \beta$, and neither $\alpha$ nor $\beta$ is zero, the element $a$ $=(\alpha, \beta)$ satisfies the equation

$$
e=(\alpha \beta)^{-1}\left\{(\alpha+\beta) a-\alpha^{2}\right\},
$$

so that $a^{-1}=(\alpha \beta)^{-1}\{(\alpha+\beta) e-a\}$. It is clear that $a^{-1}$ cannot be expressed as a polynomial of lower degree (a constant multiple of $e$ in this case).

DEFINITION 4. The range of values of $f_{J}(a)$ as $J$ varies over all maximal $B$-ideals of $A$ is the $B$-spectrum of $a$, denoted $\sigma_{B}^{\prime}(a)$. The range of values of $f_{J}(a)$ as $J$ varies over all maximal ordinary $B$-ideals of $A$ is the $B$-spectroid of $a$, denoted $\tau_{B}^{\prime}(a)$. We write $\sigma_{B}(a)=\sigma_{B}^{\prime}(a) \cap K$, and $\tau_{B}(a)=\tau_{B}^{\prime}(a) \cap K$; these may be referred to as the finite parts of the respective sets.

The set $\sigma_{B}(a)$ consists of those scalars $\alpha$ such that $a-\alpha e$ has no inverse in $B$; the set $\tau_{B}(a)$ consists of those scalars $\alpha$ such that $a$ $-\alpha e$ has no inverse in $B_{2}$. In general if $D$ is any subset of $A$, we shall denote by $\sigma_{D}(a)$ the set of scalars $\lambda \in K$ such that $(a-\lambda e)^{-1}$ fails to exist in $D$. It is clear that neither $\sigma_{B}^{\prime}(a)$ nor $\tau_{B}^{\prime}(a)$ can be empty, although each set may consist of the element $\infty$ only; an example of this is easily found in the algebra $A$ of formal power-series in an indeterminate, with $B$ the sub-algebra of series with nonnegative powers only. Here there is a unique maximal $B$-ideal, which is ordinary, consisting of series with positive powers only; if $J$ is this $B$-ideal, and $a$ $\notin B$, then clearly $f_{J}(a)=\infty$.

Since every maximal ordinary $B$-ideal of $A$ is contained in a maximal $B$-ideal, it follows that $\sigma_{B}^{\prime}(a) \supseteqq \tau_{B}^{\prime}(a)$ for all $a \in A$. The following lemma describes a case in which the two sets are equal:

Proposition 2. If $B$ is strongly saturated then $\sigma_{B}^{\prime}(a)=\tau_{B}^{\prime}(a)$ for all $a \in A$.

Proof. It is clear, in view of the remarks following Definition 4, that if $B=B_{2}$ then $\sigma_{B}(a)=\tau_{B}(a)$. If $B=B_{2}$ and $\infty \notin \tau_{B}^{\prime}(a)$ then by definition $a \in B_{2}=B$, and so $\infty \notin \sigma_{B}^{\prime}(a)$, in view of the assumption on $B$ made after Lemma 4. In view of the relation $\sigma_{B}^{\prime}(a) \supseteqq \tau_{B}^{\prime}(a)$, this completes the proof.

It is of course, not true that if $B=B_{2}$ then the maximal ordinary 
$B$-ideals coincide with the maximal $B$-ideals consider the algebra of $\S 1$, Example (iii).

Suppose that

$$
\left(a-\alpha_{1} e\right)^{-1}, \cdots,\left(a-\alpha_{r} e\right)^{-1}
$$

exist in $A$. Then if

$$
q(a)=\left(a-\alpha_{1} e\right)^{t_{1}} \cdots\left(\alpha-\alpha_{r} e\right)^{t} r,
$$

where $t_{1}, \cdots, t_{r}$ are positive integers, and $p(a)$ is any polynomial, the rational function $r(a)=p(\alpha) / q(a)$ certainly exists as an element of $A$. If this is so we have the 'spectral mapping theorem ':

THEOREM 4. If the rational function $r(a)$ of a exists in $A$ then the $B$-spectroid of $r(a)$ is the image under $r($.$) of the B$-spectroid of $a$; that is, $\alpha \in \tau_{B}^{\prime}(\alpha)$ if and only if $r(\alpha) \in \tau_{B}^{\prime}(r(a)),\left(\alpha \in K^{\prime}\right)$.

Proof. This follows at once from Theorem 2.

Notice that the spectroid, not the spectrum, is involved; the result is false in general if 'spectrum' is substituted for 'spectroid.'

CoRollary. A necessary and sufficient condition that the rational function $r(a)$ should exist as an element of $B_{2}$ is that $r\left(\tau_{B}^{\prime}(a)\right) \leqq K$.

Proof. If

$$
r(a)=\Pi\left(a-\alpha_{i} e\right)^{\rho_{i}},
$$

then

$$
\Pi\left(f_{\mathcal{J}}(\alpha)-\alpha_{i} e\right)^{\rho_{i}} \in K
$$

for all maximal ordinary $J$. Thus if $\rho_{i}<0$ we cannot have $f_{J}(\alpha)=\alpha_{i}$, and so $\left(a-\alpha_{i} e\right)^{\rho_{i}}$ exists (in $B_{2}$ and, a fortiori, in $A$ ) for all $i$ with $\rho_{i}<0$. Thus $r(a)$ exists in $A$ and the result follows at once from the theorem.

THEOREM 5. If $a$ and $a^{\prime}$ are any elements of $A$, then

$$
\tau_{B}\left(\alpha \alpha^{\prime}\right) \leqq \tau_{B}(\alpha) \cdot \tau_{B}\left(\alpha^{\prime}\right) \text { and } \tau_{B}\left(\alpha+\alpha^{\prime}\right) \subseteq \tau_{B}(\alpha)+\tau_{B}\left(a^{\prime}\right) .
$$

These relations are also true when $\tau$ is replaced by $\tau^{\prime}$, provided that the sets which occur on the right-hand sides do not contain a product $0 \cdot \infty$ or a sum $\infty+\infty$, respectively.

Proof. This also follows at once from Theorem 2. 
Theorem 5 can of course be extended to several elements of $A$, and combined with Theorem 4 to give information about the spectroid of a rational function of several elements of $A$.

Next, a condition that the spectrum should consist of the whole of $K:$

Proposition 3. If $\infty \notin \sigma_{B}^{\prime}(a)$ (that is, $\left.a \in B_{2}^{\prime}\right)$ and $a \notin B$, then $\sigma_{B}^{\prime}(a)$ $=K$.

Proof. Suppose that $\alpha \in K$ is not in $\sigma_{B}^{\prime}(\alpha)$. Then $f_{J}(\alpha-\alpha e)$ is never zero, for any maximal $B$-ideal $J$; hence, by Theorem 3 (i), $(a-\alpha e)^{-1}$ exists in $B$. Since $f_{J}(\alpha-\alpha e)$ is never $\infty$, it follows that $f_{J}\left((\alpha-\alpha e)^{-1}\right)$ is never zero. This implies that $\left((\alpha-\alpha e)^{-1}\right)^{-1}=(\alpha-\alpha e)$ is in $B$, and hence that $a \in B$.

Proposition 4. Let $\alpha \in K$ be such that $(\alpha-\alpha e)^{-1} \in B$. Then either $a \in B$, or $\infty \in \tau_{B}^{\prime}(a)$.

Proof. Suppose that $a \notin B$. The set $(a-\alpha e)^{-1} B$ is clearly an ideal of $B$; it is admissible, since $(a-\alpha e)^{-1} b=e$ would imply $a \in B$, which is not so. Hence, by Lemma 1 (ii) there is a maximal ordinary $B$-ideal, $J$ say, containing this set. Then we must have $f_{J}(a)=\infty$; for $(\alpha-\beta e) \in J$ would imply $(\alpha-\beta e)(\alpha-\alpha e)^{-1} \in J$, that is, $e+(\alpha-\beta)(\alpha-\alpha e)^{-1} \in J$, that is, $e \in J$, which is impossible.

Note that it is possible to have $a \notin B, \infty \notin \tau_{B}^{\prime}(a)$-consider Example (iii) of $\S 1$. In this case, of course, if $\alpha \notin B$ there is no $\alpha \in K$ such that $(a-\alpha e)^{-1} \in B$.

Proposition 5. If, for each $a \in A$, there exists $\alpha \in K$ such that $(a-\alpha e)^{-1} \in B$, then

(i) $B$ is strongly saturated, and

(ii) each maximal ideal of $B$ is contained in a unique maximal ordinary $B$-ideal of $A$.

Proof. (i) This follows at once from Proposition 4.

(ii) Suppose that $M$ is a maximal ideal of $B$, contained in two distinct maximal ordinary $B$-ideals of $A, J$ and $J^{\prime}$. Let $a \in A$ be such that $f_{J}(\alpha) \neq f_{J^{\prime}}(\alpha)$, and $\alpha \in K$ such that $b=(\alpha-\alpha e)^{-1}$ is in $B$. Then

$$
f_{M}(b)=f_{J}(b)=\left(f_{J}(a)-\alpha\right)^{-1} \neq\left(f_{J}(a)-\alpha\right)^{-1}=f_{J}(b)=f_{M}(b),
$$

which is a contradiction. 
5. The B-radical, semi-simplicity, etc. The theory given in this section is based on the definition of the $B$-radical of $A$ as the intersection of all maximal ordinary $B$-ideals of $A$. There is, of course, a parallel theory based on the definition of the radical as the intersection of all maximal $B$-ideals; this set is a $B$-ideal but not in general an ordinary $B$-ideal. The two theories resemble each other so closely that there seems to be no point in writing out both sets of results explicitly.

Definition 5. The intersection of all maximal ordinary $B$-ideals of $A$ is the $B$-radical of $A$.

It is evident that the $B$-radical is an ordinary $B$-ideal.

Proposition 6. If $\tau_{B}^{\prime}(a)=\{0\}$ implies $a \in B$ (in particular, if $B$ is strongly saturated) then the $B$-radical of $A$ consists of theose elements $b \in B$ such that $(e-\alpha b)$ has an inverse in $B$ for all $\alpha \in K$.

Proof. If $a$ is in the $B$-radical then $\tau_{B}^{\prime}(a)=\{0\}$, and $a \in B$, by assumption. If $e-\alpha a$ had no inverse in $B$, then $(e-\alpha a) B$ would be a proper ideal of $B$, and would be contained in a maximal ordinary $B$ ideal of $A$, by Lemma 1 (ii). If $J$ is this $B$-ideal then $f_{J}(e-\alpha a)=0$, hence $f_{J}(\alpha)=\alpha^{-1} \neq 0$, a contradiction. So $e-\alpha a$ has an inverse in $B$ for each $\alpha \in K$.

On the other hand, if $a \in B$, and $a$ is not in the $B$-radical, there will be a nonzero $\alpha \in K$ such that $f_{J}(\alpha)=\alpha$ for some $J$. Then we cannot have $\left(e-\alpha^{-1} a\right)^{-1} \in B$; if this were so then $e=\left(e-\alpha^{-1} a\right) .\left(e-\alpha^{-1} a\right)^{-1} \in J B \leqq J$, which is impossible.

Definition 6 . If the $B$-radical of $A$ is $\{0\}$ then $A$ is $B$-semi-simple. If, whenever $a \neq a^{\prime}$ there is a maximal ordinary $B$-ideal $J$ such that $f_{J}(a) \neq f_{J}\left(a^{\prime}\right)$, then $A$ is completely $B$-semi-simple.

In the case of a Banach algebra, semi-simplicity implies complete semi-simplicity. Whether this is so in the present more general case remains an open question. We shall obtain partial results in this direction under restrictive hypotheses.

Proposition 7. If $A$ is $B$-semi-simple, $a \neq a^{\prime}$, and $\tau_{B}^{\prime}(a)$ is not the whole of $K^{\prime}$, then there is a maximal ordinary $B$-ideal $J$ such that $f_{J}(a)$ $\neq f_{J}\left(a^{\prime}\right)$.

Proof. If $f_{J}(a)$ never takes the value $\infty$, then clearly $f_{J}(a)=f_{J}\left(a^{\prime}\right)$ for all maximal ordinary $J$ implies $a=a^{\prime}$, by the definition of $B$-semisimplicity. 
If $f_{J}(a)$ never takes the value $\alpha$, then by Theorem $3,(a-\alpha e)^{-1} \in B_{2}$. If $f_{J}(a)=f_{J}\left(a^{\prime}\right)$ for all $J$ then $f_{J}\left((a-\alpha e)^{-1}\right)=f_{J}\left(\left(a^{\prime}-\alpha e\right)^{-1}\right)$ for all $J$, whence $(a-\alpha e)^{-1}=\left(\alpha^{\prime}-\alpha e\right)^{-1}$, by the assumed $B$-semi-simplicity. Hence $a-\alpha e=a^{\prime}-\alpha e$, and $a=a^{\prime}$.

Lemma 10. If $A$ is $B$-semi-simple, and $\tau_{B}^{\prime}(a)$ contains no nonzero elements of $K$, then $a=0$.

Proof. Since $K$ is assumed to be algebraically closed, there will be in $K$ an element different from 0 and from 1 ; let $\alpha$ be any such element. Since $f_{J}(\alpha)$ is never $1,(a-e)^{-1}$ exists in $B_{2}$, by Theorem 3. Similarly, $(\alpha a-e)^{-1}$ exists in $B_{2}$. Clearly $f_{J}\left((a-e)^{-1}\right)=f_{J}\left((\alpha a-e)^{-1}\right)$ for all $J$ $\left(=0\right.$ if $f_{J}(a)=\infty,=-1$ if $\left.f_{J}(a)=0\right)$. Hence $(a-e)^{-1}=(\alpha a-e)^{-1}$, whence $a-e=\alpha a-e$, giving $a=0$.

Lemma 11. If $\tau_{B}^{\prime}(a)$ contains a finite number of elements of $K$ only, then it does not contain $\infty$, if $A$ is $B$-semi-simple.

Proof. Let $\alpha_{1}, \alpha_{2}, \cdots, \alpha_{n}$ be the elements of $K$ in $\tau_{B}^{\prime}(\alpha)$. Then $f_{J}\left(\left(a-\alpha_{1} e\right)\left(a-\alpha_{2} e\right) \cdots\left(a-\alpha_{n} e\right)\right)=0$ or $\infty$ only. By Lemma 10 this implies that $\left(a-\alpha_{1} e\right)\left(a-\alpha_{2} e\right) \cdots\left(\alpha-\alpha_{n} e\right)=0$, and this is clearly inconsistent with $f_{J}(a)=\infty$ for any $J$.

COROLlaRY. If there are only a finite number of maximal ordinary $B$-ideals, then $f_{J}(a)$ is never $\infty$ for any $a \in A$, that is, $A=B_{2}$.

It is clear that if we know that for each $a \in A, f_{J}(\alpha)=\infty$ for a finite set of maximal ordinary $B$-ideals only, simplifications will result.

Definition 7. The algebra $A$ is of finite type (with respect to $B$ ) if for each $a \in A$ the function $f_{J}(a)$ is infinite on (at most) a finite set of maximal ordinary $B$-ideals $J$.

The algebra of rational functions of an indeterminate is evidently of finite type with respect to the sub-algebra of constants.

Proposition 8. If $A$ is $B$-semi-simple and of finite type, it is completely B-semi-simple.

Proof. Suppose that $f_{J}(a)=f_{J}\left(a^{\prime}\right)$ for all $J$. Then $f_{J}\left(a-a^{\prime}\right)$ takes a finite set of nonzero values at most. Hence, by Lemma 11, $f_{J}\left(a-a^{\prime}\right)$ is never $\infty$. If $a \neq a^{\prime}$, then $a\left(a-a^{\prime}\right)$ would be such that (i) $f_{J}\left(a\left(a-a^{\prime}\right)\right)$ $=\infty$ for some $J$; and (ii) $f_{J}\left(a\left(a-a^{\prime}\right)\right)$ takes a finite set of values in $K$ 
only-two contradictory properties. Hence $a=a^{\prime}$.

There are two problems which are closely related to each other and to $B$-semi-simplicity. These are, broadly speaking, (i) on how large a set of maximal ordinary $B$-ideals can the function $f_{J}(a)$ be $\infty$ ? and (ii) on how small a set can $f_{J}(a)$ be nonzero, with $a \neq 0$ ? In the absence of $B$-semi-simplicity, of course, $f_{J}(a)$ may be $\infty$ for all maximal ordinary $J$, and $f_{J}\left(a^{\prime}\right)$ may be zero for all such $J$, with $a^{\prime} \neq 0$ (consider the example of formal power-series discussed after Definition 4).

In the next two propositions we assume that $A$ is $B$-semi-simple.

Proposition 9. Let $\mathscr{l l}$ be a finite set of maximal ordinary $B$ ideals, and $a \in A$ an element such that $f_{J}(a)=\infty$ for $J \in \mathscr{l l}$, and $f_{J}(a)$ $\neq \infty$ for $J \notin \mathscr{M l}$. Then, if $f_{J}\left(\alpha^{\prime}\right)=0$ for all $J \notin \mathscr{l l}$, it follows that $a^{\prime}=0$.

Proof. The function $f_{J}\left(\alpha \alpha^{\prime}\right)$ takes a finite set of values only, hence it is never $\infty$, by Lemma 11 . This clearly implies that $f_{J}\left(a^{\prime}\right)=0$ for all $J$, and so $a^{\prime}=0$, by the assumed $B$-semi-simplicity.

A somewhat similar result is the following.

Proposition 10. Let $\mathscr{l l}$ be a set of maximal ordinary B-ideals such that there is an element $a \in A$ with $f_{J}(a)=\infty$ for $J \in \mathbb{M l}$, and $f_{J}(a)$ $\neq \infty$ for $J \notin \mathscr{A l}$. Then there is no element $a^{\prime} \in A$ with $f_{J}\left(a^{\prime}\right) \neq 0$ for $J \in \mathscr{M}$, and $f_{J}\left(a^{\prime}\right)=0$ for $J \notin \mathscr{l}$.

Proof. If there were such an element $a^{\prime}$ then we would have $f_{J}\left(a a^{\prime}\right)=0$ or $\infty$ only, whence $a a^{\prime}=0$, by Lemma 10 . This contradicts $f_{J}\left(a a^{\prime}\right)=\infty$ for $J \in \mathscr{M}$.

If $A$ is $B$-semi-simple, then Theorem 2 states that $A$ is isomorphic' in a certain sense to an algebra of functions on the set of maximal ordinary $B$-ideals. In certain cases it is possible to assert that there is a genuine isomorphism between $A$ and an algebra of equivalenceclasses of functions. We introduce this as follows.

Let $X$ be any set. We shall call a family $Q^{\prime}$ of subsets of $X$ a $Q$-family if (i) the union of two (and hence any finite number of) subsets of $Q$ is in $Q$; (ii) $X$ is not in $Q$. For example, if $X$ is the real interval $(0,1)$, the subsets of measure zero form a $Q$-family. Take now the set $S$ of functions defined on $X$, with values in $K^{\prime}$, which are finite outside a set of $\mathscr{Q}$. Let $T$ be the set of functions which are zero 
outside a set of $Q$. Let $(S ; T)$ be the set of equivalence-classes of functions of $S$, modulo functions of $T$. Then, in the familiar way, $(S ; T)$ can be made into an algebra by defining the sum of two classes to be the class determined by the sum of two functions, one from each class, etc.; it is easy to verify that the algebraic operations are well-defined. The object of condition (ii) is to ensure that the resulting algebra is nontrivial.

Definition 8. If $X$ is the set of maximal ordinary $B$-ideals of $A$, and there is a $Q$-family of subsets of $X$ such that $A$ is isomorphic to $(S ; T)$, as defined above, then $A$ has a Q-representation.

THEOREM 6. If $A$ is B-semi-simple, and of finite type, it has a $Q$ representation.

Proof. If there are finitely many maximal ordinary $B$-ideals, then $f_{J}(a)$ is always finite, by Lemma 11 , Corollary. Then result follows at once in this case, taking the $Q$-family consisting of the empty set only.

If there are infinitely many maximal ordinary $B$-ideals, then it is easy to verify that the family of sets on which $f_{J}(a)$ is infinite for some $a \in A$ forms a $Q$-family. The required result then follows from Proposition 9.

It would be of considerable interest to extend the above results, in particular, to remove the qualification 'finite' from the set $\mathscr{C}$ in Proposition 9. In $\S 7$ we shall do this under additional hypotheses (Proposition 17). It is not evident that these restrictions are necessary for the validity of the result, and more information on the point would be welcome. There is one partial result in this direction, as follows:

Proposition 11. If $A$ is completely $B$-semi-simple, then $f_{J}(a)=\infty$ for $J \in \mathscr{l}, f_{J}\left(a^{\prime}\right)=0$ for $J \notin \mathscr{K}, a^{\prime} \in B$ together imply $a^{\prime}=0$.

\section{Proof. Immediate.}

6. Algebras over topological fields. We now consider the case of a field $K$ with a topology. We are primarily interested in the complex case, but it is as easy to write out the results for much more general fields. We require very little of the topology; the essential feature is that it should provide a reasonable definition of 'bounded' subsets of $K$. We shall assume (until after Proposition 15 ) that $K$ is a topological 
field in the sense of Bourbaki, that is, that the topology is Hausdorff and the algebraic operations are continuous.

We adopt the definition of boundedness given by Shafarevich; the subset $H$ of $K$ is bounded if, given any neighborhood $N$ of 0 , there is a neighborhood $N^{\prime}$ of 0 such that $H N^{\prime} \leqq N$. It is trivial that the union, sum and product of two bounded subsets of $K$ are again bounded subsets. We shall further assume (again until after Proposition 15) that $K$ is of type $V$, in the sense of Kaplansky; that is, if the set $S$ is disjoint from some neighborhood of 0 , then the set of inverses $S^{-1}$ is bounded. We assume that $K$ is not discrete; if $K$ is discrete then every subset of $K$ is closed and bounded, and the results reduce to those of $\S 4$.

Definition 9. Denote by $B_{1}$ the set of elements of $A$ which have a bounded $B$-spectroid, and by $B_{1}^{\prime}$ the set of elements with a bounded $B$-spectrum. If $B_{1} \supseteqq B$ then $B$ is weakly bounded; if $B_{1}^{\prime} \supseteqq B$ then $B$ is strongly bounded. If $B_{1} \subseteq B$, then $B$ is strongly boundedly saturated; if $B_{1}^{\prime} \subseteq B$ then $B$ is weakly boundedly saturated.

It is evident that $B_{1} \supseteq B_{1}^{\prime} ; B_{1}$ is clearly a sub-algebra of $A$, by Lemma 3 , but $B_{1}^{\prime}$ is not a sub-algebra of $A$ in general.

For the remainder of this section we shall assume that $B$ is weakly bounded, unless the contrary is explicitly stated.

Proposition 12. (i) The maximal ordinary $B_{1}$-ideals of $A$ are the same as the maximal ordinary $B$-ideals.

(ii) If $M$ is any maximal ideal of $B_{1}$, then $B_{1} / M \cong K$.

(iii) $\left(B_{1}\right)_{1}=B_{1}$, for any $B$.

Proof. This is analogous to that of Proposition 1.

It is always possible, for any given $A$, to chose a strongly bounded sub-algebra $B$; take $B=K e$. Also, it is always possible to choose a strongly boundedly saturated $B$; take $B=(K e)_{1}$.

If $B$ is not weakly bounded, there may be $B_{1}$-ideals of $A$ which are not $B$-ideals. For example, let $I$ be any infinite index-set, and $A$ the algebra of complex-valued functions defined on $I,\left\{a_{i}\right\}_{i \in I}$, with pointwise addition and multiplication. Take $B=A$; then $B_{1}$ is the set of all bounded functions on $I$. Any function $a \in A$ with $a_{i} \neq 0$ for all $i \in I$, but $\inf _{i \in I}\left|a_{i}\right|=0$, will be in an ordinary $B_{1}$-ideal but in no proper $B$-ideal of $A$.

Lemma 12. If 0 adheres to $\sigma_{B}^{\prime}(a)$ then $0 \in \sigma_{B}^{\prime}(a)$; if 0 adheres to 
$\tau_{B}^{\prime}(a)$ then $0 \in \tau_{B}^{\prime}(a)$

Proof. We shall prove the second statement only; the proof of the first is similar and slightly simpler. The set $a B+a^{2} B+a^{3} B+\cdots$ is clearly an ordinary $B$-ideal of $A$. If $e$ were in this $B$-ideal, then

$$
e=b_{1} a+b_{2} a^{2}+\cdots+b_{n} a^{n}
$$

for some $b_{1}, b_{2}, \cdots, b_{n} \in B$. It is elementary to verify that if 0 adheres to $\tau_{B}^{\prime}(a)$ then it adheres to $\tau_{B}^{\prime}\left(b_{1} a+\cdots+b_{n} a^{n}\right)$ also. Thus it would adhere to $\tau_{B}^{\prime}(e)=\{1\}$. This is impossible, since the topology of $K$ is Hausdorff. So the $B$-ideal specified above is admissible, and there is a maximal ordinary $B$-ideal, $J$ say, containing it. Thus $a=a e \in J$, and $f_{J}(a)=0$, so that $0 \in \tau_{B}^{\prime}(\alpha)$, as asserted.

THEOREM 7. For each $a \in A, \sigma_{B}(a)$ and $\tau_{B}(a)$ are closed subsets of $K$.

Proof. If $\alpha \in K$ adheres to $\sigma_{B}(a)$ then clearly 0 adheres to $\sigma_{B}(a$ $-\alpha e$ ); hence, by Lemma 12,0 is in $\sigma_{B}(\alpha-\alpha e)$ and so $\alpha$ is in $\sigma_{B}(\alpha)$. Similarly for $\tau_{B}(a)$.

We may topologise $K^{\prime}$ by taking the basic neighborhoods of $\infty$ to be the complements in $K^{\prime}$ of the bounded subsets of $K$. In this topo$\operatorname{logy}, \tau_{B}^{\prime}(a)$ and $\sigma_{B}^{\prime}(a)$ are not in general closed in $K^{\prime}$. Example (iii) of $\S 1$ shows that we may have $\infty$ adherent to $\tau_{B}^{\prime}(a)$, but no maximal ordinary $J$ such that $f_{J}(a)=\infty$.

Theorem 8. If $a$ is in no maximal ordinary $B$-ideal of $A$ then $a$ has an inverse in $B_{1}$.

Proof. (1) As in Theorem 3 (ii), $a$ has an inverse $a^{-1}$ in $A$. Since $f_{J}(a)$ is never zero, there is a neighborhood $N$ of 0 such that $N \cap \tau_{B}^{\prime}(a)$ is empty, by Theorem 7. Since we assume that $K$ is of type $V$, this implies that the set of inverses of elements of $\tau_{B}^{\prime}(a)$ is bounded; but this set of inverses is evidently the $B$-spectroid of $a^{-1}$; hence $a^{-1} \in B_{1}$.

COROLlaRY. If $B$ is strongly boundedly saturated, then $a \in A$ has an inverse $a^{-1} \in B$ if and only if $a$ is in no maximal ordinary B-ideal of $A$.

Proposition 13. If $a \in B_{2}, a \notin B$, and $B$ is strongly boundedly saturated, then $\tau_{B}^{\prime}(a)=K$. 
Proof. This follows from Theorem 8, Corollary, just as Proposition 3 follows from Theorem 3 (i).

We note that if $B$ is strongly boundedly saturated, the hypothesis of Proposition 6 is satisfied, and hence the conclusion of the proposition is valid.

Proposition 14. If $p(a) \in B_{1}$ for some polynomial $p$ of degree $\geqq 1$, then $a \in B_{1}$.

Proof. Since $\tau_{B}^{\prime}(p(\alpha))$ is bounded, we can choose $\alpha \in K$ so that $0 \notin \tau_{B}^{\prime}(q(a))$, where $q(a)=p(a)-\alpha e$. Thus $q(a) \in B_{1}$ and $(q(a))^{-1}$ exists. Write $(q(a))^{-1}$ as a sum of terms of the type $\beta_{r s}\left(\alpha-\alpha_{r} e\right)^{-s}$, it is a matter of routine to verify that if $\tau_{B}^{\prime}(\alpha)$ is unbounded, then 0 adheres to $\tau_{B}^{\prime}\left((q(a))^{-1}\right)$. This contradicts the fact that $q(a) \in B_{1}$, and $\tau_{B}^{\prime}\left((q(a))^{-1}\right)$ $=\left\{\tau_{B}^{\prime}(q(a))\right\}^{-1}$.

The corresponding result, with $B_{2}$ in place of $B_{1}$, is true and trivial.

The following result is analogous to Theorem 4, Corollary, and is proved in exactly the same way:

Proposition 15. A necessary and sufficient condition that the rational function $r(a)$ exists as an element of $B_{1}$ is that $r\left(\tau_{B}^{\prime}(a)\right)$ is a bounded subset of $K$.

For the remainder of this section we assume only that the field $K$ has a Hausdorff topology, and that addition is continuous. We may topologise $K^{\prime}$ by taking the neighborhoods of $\infty$ to be the complements in $K^{\prime}$ of the bounded subsets of $K$. It is possible to introduce a topology on the maximal ordinary $B$-ideals of $A$ in at least three obvious ways:

(i) Take as basic neighborhoods of the maximal ordinary $B$-ideal $J_{0}$ the sets $\left\{J: f_{J}\left(a_{r}\right) \in N, r=1,2, \cdots, n\right\}$, where $N$ is any neighborhood of 0 and $a_{1}, a_{2}, \cdots, a_{n}$ are any elements of $J_{0}$. This clearly defines a Hausdorff topology in which each function $f_{J}(a)$ is continuous (as a function of $J$ ) wherever it is finite. In particular all functions representing elements of $B_{2}$ are continuous everywhere.

(ii) Take as basic neighborhoods of $J_{0}$ the sets

$$
\left\{J: f_{J}\left(a_{r}\right) \in N_{r}\left(f_{J_{0}}\left(a_{r}\right)\right), r=1,2, \cdots, n\right\},
$$

where $a_{1}, a_{2}, \cdots, a_{n}$ are any elements of $A$ and $N_{1}, N_{2}, \cdots, N_{n}$ are any 
neighborhoods of $f_{J_{0}}\left(a_{1}\right), f_{J_{0}}\left(a_{2}\right), \cdots, f_{J_{0}}\left(a_{n}\right)$ respectively. This is the weakest topology in which all the functions $f_{J}(a)$ are continuous. It is evidently finer than (i).

(iii) Take as basic neighborhoods of $J_{0}$ the sets $\left\{J: f_{J}\left(b_{r}\right) \in N\right.$, $r=1,2, \cdots, n\}$, where $N$ is any neighborhood of 0 and $b_{1}, b_{2}, \cdots, b_{n}$ are any elements of $J_{0} \cap B$.

Other variations are possible; for instance, $B$ may be replaced by $B_{1}$ or $B_{2}$ in (iii). We shall refer to these variations as (iii'), (iii'), respectively.

In general, topology (iii) will not be Hausdorff; a necessary and sufficient condition that it should be so is that each maximal ideal of $B$ should be contained in precisely one maximal ordinary $B$-ideal of $A$. If the topology is Hausdorff, then the set $\mathscr{J}_{0}^{\prime}$ of maximal ordinary $B$ ideals of $A$ is compact; Gelfand's proof of the corresponding result for Banach algebras [4, Satz 9] applies to the present case. Similar remarks apply to (iii') and (iii').

In the case where $A$ is a Banach algebra, and $B=A$, all the above topologies reduce to the customary Gelfand topology on the maximal ideals. In the context of $\S 8$, topology (ii) seems the most appropriate.

Similar topologies could of course be imposed on the space of all maximal $B$-ideals of $A$.

7. Self-adjoint algebras. As in $\S 5$, we use the maximal ordinary $B$-ideals; similar results could be obtained, starting from the maximal $B$-ideals. In this section the scalar field is taken to be the complex field $C$. The results could be formulated in a more general situation (in a field with a suitable 'conjugation'), but there seems to be no point in doing this. Asterisks applied to scalars denote complex conjugates, and $\infty^{*}=\infty$.

Definition 10. The algebra $A$ is self-adjoint (with respect to $B$ ) if, given $a \in A$, there exists $a^{*} \in A$ (not necessarily unique) such that $f_{J}\left(a^{*}\right)=f_{J}(a)^{*}$ for each maximal ordinary $J$.

From now on it is assumed that $A$ is self-adjoint and $B$-semisimple.

Proposition 16. The algebra $A$ is completely B-semi-simple.

Proof. Suppose that $f_{J}(a)=f_{J}\left(a^{\prime}\right)$ for all $J$. Then evidently $\left(e+a a^{*}\right)^{-1}$ and $\left(e+a^{\prime} a^{\prime *}\right)^{-1}$ both exist (in $\left.B_{1}\right)$ and $f_{J}\left(\left(e+a a^{*}\right)^{-1}\right)=f_{J}\left(\left(e+a^{\prime} a^{\prime *}\right)^{-1}\right)$ for all $J$. Hence the two inverses are equal, by the assumed $B$-semi- 
simplicity, and this implies $a a^{*}=a^{\prime} a^{\prime *}$. Next, it is easy to verify that $a\left(e+a a^{*}\right)^{-1}$ and $\alpha^{\prime}\left(e+a^{\prime} a^{*}\right)^{-1}$ are both in $B_{1}$, and $f_{J}\left(a\left(e+a a^{*}\right)^{-1}\right)=f_{J}\left(a^{\prime}(e\right.$ $\left.\left.+a^{\prime} a^{\prime *}\right)^{-1}\right)$ for all $J$. Hence the two elements are equal, and the conclusion $a=a^{\prime}$ is immediate.

COROLlary. The element $a^{*}$ is unique.

It is clear that $a^{*}=0$ implies $a=0$, and $a a^{*}=0$ implies $a=0$.

The next result is, as promised in $\S 5$, an improvement of Proposition 9 in the present special case:

Proposition 17. If $\mathscr{C l}$ is a set of maximal ordinary B-ideals and $a \in A$ is such that $f_{J}(a)=\infty$ for $J \in \mathscr{l}, f_{J}(a) \neq \infty$ for $J \notin \mathscr{L}$, then $f_{J}\left(a^{\prime}\right)$ $=0$ for $J \notin \mathcal{L l}$ implies $a^{\prime}=0$.

Proof. Since $f_{J}\left(a^{\prime} a^{*}+e\right)$ is either real and $\geqq 1$, or is infinite, it is clear that $f_{J}\left(a a^{\prime} a^{\prime *}+a\right)=f_{J}(a)$ for $J \in \mathscr{C}$; and since $f_{J}\left(a^{\prime}\right)=0$ for $J \notin \mathscr{l}$, the same equation holds for $J \notin \mathscr{C}$ also. Hence $a a^{\prime} a^{\prime *}=0$, by Proposition 16. But this implies $a^{\prime}=0$; if not, there would be a $B$-ideal $J \in \mathscr{C l}$ with $f_{J}\left(a^{\prime}\right) \neq 0$, which would imply $f_{J}\left(a a^{\prime} a^{\prime *}\right)=\infty$, which contradicts $a a^{\prime} a^{\prime *}=0$.

THEOREM 9. If $A$ is $B$-semi-simple and self-adjoint, it has a $Q$ representation.

Proof. If $f_{J}(a)=\infty$ for $J \in \mathscr{l}$, and $f_{J}\left(a^{\prime}\right)=\infty$ for $J \in \mathscr{C l}^{\prime}$, it follows that if $a^{\prime \prime}=\left(e+a a^{*}\right)\left(e+a^{\prime} a^{\prime *}\right)$ then $f_{J}\left(a^{\prime \prime}\right)=\infty$ for $J \in \mathscr{C} \cup \mathscr{C}^{\prime}$. Also, $f_{J}(a)$ cannot be infinite for all maximal ordinary $B$-ideals $J$, by Lemma 10. Hence the family of sets on which $f_{J}(a)$ is infinite for some $a \in A$ is a $Q$-family. The required result now follows from Proposition 17.

So far the topology of $C$ has not been involved; it is essential for the results which follow. From now on we suppose $B=B_{1}$, that is, $B$ is weakly bounded and strongly boundedly saturated (Definition 9). In the absence of this assumption the following results remain true, when suitably modified. But the statements then become more complicated, and the gain in generality is not significant.

Proposition 18. $B=B_{2}$. 
Proof. For any $a, f_{\mathrm{J}}\left(e+a a^{*}\right)$ is never zero, and so $\left(e+a a^{*}\right)^{-1}$ exists. If $a \in B_{2}$, then $f_{J}\left(\left(e+a a^{*}\right)^{-1}\right)$ is never zero. By Lemma 12, it is therefore bounded away from zero, and so $f_{J}(a)$ is bounded away from infinity. Thus $a \in B_{1}=B$. Since $B_{2} \supseteq B$, the theorem follows.

Corollary. $\tau_{B}^{\prime}(a)$ is closed in $C^{\prime}$ for any $a$.

LEMMA 13. Each maximal ideal of $B$ is contained in exactly one maximal ordinary $B$-ideal of $A$.

Proof. Suppose that the maximal ideal $M$ of $B$ is contained in the distinct maximal ordinary $B$-ideals, $J$ and $J^{\prime}$, of $A$. Let $a \in J, a \notin J^{\prime}$. Then

$$
f_{M}\left(\left(e+a a^{*}\right)^{-1}\right)=\left(1+\left|f_{J}(a)\right|^{2}\right)^{-1} \neq\left(1+\left|f_{J}(a)\right|^{2}\right)^{-1}=f_{M}\left(\left(e+a a^{*}\right)^{-1}\right),
$$

a contradiction.

Lemma 14. All the topologies described in $\S 6$ are equivalent.

Proof. It is clearly sufficient to prove that (iii) is finer than (ii). Let $N$ be the neighborhood

$$
N=\left\{J: f_{J}\left(a_{r}\right) \in N_{r}\left(f_{J_{0}}\left(a_{r}\right)\right), r=1,2, \cdots, n\right\} .
$$

Write

$$
b_{r}=\left(e+a_{r} a_{r}^{*}\right)^{-1}
$$

it is easy to find neighborhoods $N_{r}^{\prime}, N_{r}^{\prime \prime}$ such that

$$
f_{J}\left(b_{r}\right) \in N_{r}^{\prime}\left(f_{J_{0}}\left(b_{r}\right)\right), f_{J}\left(a_{r} b_{r}\right) \in N_{r}^{\prime \prime}\left(f_{J_{0}}\left(a_{r} b_{r}\right)\right)
$$

together imply

$$
f_{J}\left(a_{r}\right) \in N_{r}\left(f_{J_{0}}\left(a_{r}\right)\right)
$$

(if $f_{J_{0}}\left(a_{r}\right)=\infty$, then $N_{r}^{\prime \prime}$ is superfluous). By translating the neighborhoods $N_{r}^{\prime}, N_{r}^{\prime \prime}$ to the origin if necessary, and taking their intersection, it is easily seen that there is a neighborhood in topology (iii) which is contained in $N$.

Combining the above results, we obtain at once:

THEOREM 10. Let $A$ be a self-adjoint, B-semi-simple algebra, with $B=B_{1}$. Then the maximal ordinary $B$-ideals of $A$ can be topologised so as to become a compact Hausdorff space, and the mapping $a \rightarrow f_{J}(a)$ 
sends elements of $A$ into continuous $C^{\prime}$-valued functions on this space. The sets on which $f_{J}(a)$ is infinite for some $a \in A$ form a Q-family of closed sets.

It is apparent that the structure space depends (set-wise and topologically) only on the 'bounded' sub-algebra $B$ of $A$, provided that this satisfies reasonable conditions, which ensure that it is large enough. If we assume a little more, namely that all bounded continuous functions correspond to elements of $B$ (for instance, if $B$ is a Banach algebra under a suitable norm), then we can clearly assert that the set on which $f_{J}(a)$ is infinite is nowhere dense (since the set is closed, this is equivalent to its interior being empty). The conclusion of Theorem 10 is thus strengthened.

To conclude this section we turn to Example (vi) of $\S 1$, and see to what extent the results of this section can be applied to it. First, it seems desirable to state precisely what we mean by an algebra of normal operators on a Hilbert space; we mean a collection $A$ of normal operators such that any scalar multiple of an operator in $A$ is in $A$, and the sum and product of any two operators in $A$ have unique extensions in $A$. As always, we assume that $A$ contains a unit (the identity operator, here) and is commutative (in the sense that the product of two operators, in a certain order, has the same extension in $A$ as the product in the reverse order). We take $B$ to consist of the bounded operators in $A$ : we assume that if $a \in A$, and $a^{-1}$ exists as a bounded operator, then $a^{-1} \in B$, and we also assume that $B$ is uniformly closed. This implies that the maximal ideal condition $B / M \cong C$ is satisfied. If we denote by $a^{*}$ the usual Hilbert space adjoint of $a$ (we proceed immediately to show that this is in agreement with the previous use of $a^{*}$ ), and restrict attention to algebras $A$ which are self-adjoint in the sense that $a \in A$ implies $a^{*} \in A$, then we have the following.

Lemma 15. The algebra $A$ is self-adjoint in the sense of Definition 10.

Proof. If $a$ is bounded then it is clear that $f_{J}\left(a+a^{*}\right)$ is real, since $\left(a+a^{*}-\lambda e\right)$ has an inverse in $B$ for nonreal $\lambda$. Similarly, $f_{J}\left(a-a^{*}\right)$ is imaginary, and so $f_{J}\left(a^{*}\right)=f_{J}(a)^{*}$ for bounded $a$. Next, for any $a \in A$, write $b=\left(e+a a^{*}\right)^{-1}$; it is well known that $b \in B$ and $a b \in B$; also $b$ is self-adjoint $\left(b^{*}=b\right)$, and $(a b)^{*}=a^{*} b$. If $f_{J}(a)$ and $f_{J}\left(a^{*}\right)$ are both finite, then from $f_{J}\left(a^{*} b\right)=f_{J}(a b)^{*}$ it follows that $f_{J}\left(a^{*}\right)=f_{J}(a)^{*}$, since $f_{J}(b)$ is real and nonzero. It remains to show that if one of $f_{J}(a), f_{J}\left(a^{*}\right)$ is finite, then so is the other. Suppose the contrary; there is no loss of generality in supposing $f_{J}(a)=1, f_{J}\left(a^{*}\right)=\infty$. Then 


$$
1=f_{J}(e-b)=f_{J}\left(a a^{*} b\right)=f_{J}(a) f_{J}\left(a^{*} b\right)=f_{J}(a) f_{J}(a b)^{*}=0,
$$

which is impossible.

The sub-algebra $B$ is semi-simple, by the usual reasoning, and the $B$-semi-simplicity of $A$ follows at once from this. The conclusions of Lemma 13 and 14 are true, independently of any assumption that $B$ $=B_{1}$, since $b=\left(e+a a^{*}\right)^{-1}$ and $a b$ are certainly in $B$.

The conclusion of Theorem 10 is thus valid for $A$. Moreover, the assumption that $B$ is uniformly closed ensures that the functions $f_{J}(a)$ become infinite only on nowhere dense sets. The fact that $B=B_{1}$ follows from the same assumption; for each bounded continuous function on the maximal $B$-ideals of $A$ (=maximal ideals of $B$ ) corresponds to an element of $B$.

In the paper of Fell and Kelley [3], the authors deal with algebras of operators from a somewhat different point of view. Starting from a strongly closed algebra of bounded of bounded operators, they select a class of unbounded functions on the structure space (the same class as we have obtained above, namely the continuous $C^{\prime}$ valued functions infinite only on a nowhere dense set), and show that to each such function there corresponds a normal operator. Every normal operator can be obtained in this way, starting from a suitable algebra of bounded operators. The problem of the functional representation of an algebra of operators is not explicitly treated.

As a realization of the sort of algebra we have been considering, take the following trivial example. Let the Hilbert space be $L_{2}(0,1)$, and consider continuous $C^{\prime}$-valued functions on $(0,1)$ which are infinite only on a set with empty interior. To each such function a normal operator can be attached in an obvious way; the operator, applied to a function of $L_{2}$, yields the ordinary product of the two functions. If we assume that $A$ is an algebra of such operators, containing all operators corresponding to bounded functions, then the above theory can be applied, and it is found that the operators are represented by the functions from which they have arisen.

8. Algebraic function fields. Although it is not our main objective, we give a few indications of the relation between the theory developed in the preceding sections, and the theory of fields of algebraic functions of one variable. All the relevant definitions, etc., will be found in Chevalley's book [2]. The first result is valid quite generally.

Lemma 16. If $A$ is a field, and $K$ is a proper sub-field of $A$, then for every maximal ordinary $K$-ideal $J$ of $A$ there is an element $a \in A$ with $f_{J}(a)=\infty$. 
Proof. If not, $J$ would be a proper ideal of $A$, different from $\{0\}$, by Lemma 3 ; this is impossible.

The definition of a $V$-ring, as required in the next lemma, will be found in $[2, p .1]$.

THEOREM 11. If $A$ is a field of algebraic functions of one variable and $K$ is an algebraically closed proper sub-field of $A$, then the maximal ordinary $K$-ideals of $A$ are in one-to-one correspondence with the $V$ rings in $A$ (over $K$ ).

Proof. Let $J$ be a maximal ordinary $K$-ideal of $A$, and write $Q$ $=J+K$. Then clearly $Q$ is a ring; further (i) $Q$ contains $K$; (ii) $Q \neq A$, since, by Lemma 16 there is an element $a \in A$ with $f_{J}(a)=\infty$; and $f_{J}(q)$ $\in K$ for all $q \in Q$; (iii) if $x \notin Q$ then $f_{J}(x)=\infty$; for if $f_{J}(x)=\alpha \in K$ then $x-\alpha e \in Q$ and so $x \in Q$. If $f_{J}(x)=\infty$ then $f_{J}\left(x^{-1}\right)=0$, by Lemma 9 , and so $x^{-1} \in Q$. Thus $Q$ is a $V$-ring.

On the other hand, let $Q$ be any $V$-ring, and let $J$ be the ideal of non-units. Then $Q / J \cong K([2]$, p. 10$)$; every element of $Q$ is of the form $\alpha e+j$, where $j \in J$. Clearly $J$ is an ordinary $B$-ideal; we now show that it is maximal. Let $a$ be any element of $A$, not in $J$. Then if $a \in Q, a-\alpha e \in J$ for some $\alpha \in K$ and so $e$ is in the $K$-ideal generated by $J$ and $a$. If $a$ is not in $Q$ then $a^{-1}$ must be in $J$; for if $a^{-1}$ were in $Q$ but not in $J$ then $\alpha^{-1}$, and hence $a$, would be a unit in $Q$. So again $e$ is in the $K$-ideal generated by $J$ and $a$. That is, $J$ is maximal. This establishes the required correspondence.

We may thus identify the maximal ordinary $K$-ideals in $A$ with the places of $A$, where $A$ is a field of algebraic functions of one variable over $K$. The value taken by $a \in A$ at the place $J[2, \mathrm{p} .6]$ is the same as the value of the function $f_{J}(a)$ as defined in $\S 2$.

The places of $A$ may be topologised, if $K$ is a topological field; the topology (ii) previously indicated $(\S 6)$ reduces to that given by Chevalley for the complex case [2, p. 133].

\section{REFERENCES}

1. R. Arens, A generalization of normed rings, Pacific J. Math., 2 (1952), 455-471.

2. C. Chevalley, Algebraic functions of one variable, New York, 1951.

3. J. M. G. Fell and J. L. Kelley, An algebra of unbounded operators, Proc. Nat. Acad. Sci. 38 (1952), 592-598.

4. I. M. Gelfand, Normierte Ringe, Mat. Sbornik, N. S. 9 (51) (1941) 3-24.

5. I. Kaplansky, Topological rings, Amer. J. Math. 69 (1947), 153-183.

6. E. A. Michael, Locally multiplicatively-convex topological algebras, (Amer. Math. Soc. Memoir no. 11) 1952. 
7. M. H. Stone, Linear transformations in Hilbert space, New York 1932.

8. L. Waelbroeck, Le calcul symbolıque dans les algèbres commutatives, C. R. Acad. Sci. Paris 238 (1954), 556-558, 640-641, 762-764.

9. - Le calcul symbolique dans les algèbres commutatives, J. Math. Pures Appl. N. S. 33 (1954), 147-186.

The Institute for Advanced Study, Princeton

AND QUEEN'S UNIVERSITY, BELFAST 



\section{PACIFIC JOURNAL OF MATHEMATICS}

\section{EDITORS}

H. L. ROYDEN

Stanford University

Stanford, California

R. A. Beaumont

University of Washington

Seattle 5, Washington

\section{A. L. Whiteman}

University of Southern California

Los Angeles 7, California

E. G. Straus

University of California

Los Angeles 24, California

\section{ASSOCIATE EDITORS}
E. F. BECKENBACH
C. E. BURGESS
M. HALL
E. HEWITT

\author{
A. HORN \\ V. GANAPATHY IYER \\ R. D. JAMES \\ M. S. KNEBELMAN
}

L. NACHBIN

I. NIVEN

G. SZEKERES

T. G. OSTROM

M. M. SCHIFFER
F. WOLF

K. YOSIDA

\section{SUPPORTING INSTITUTIONS}

\author{
UNIVERSITY OF BRITISH COLUMBIA \\ CALIFORNIA INSTITUTE OF TECHNOLOGY \\ UNIVERSITY OF CALIFORNIA \\ MONTANA STATE UNIVERSITY \\ UNIVERSITY OF NEVADA \\ OREGON STATE COLLEGE \\ UNIVERSITY OF OREGON \\ UNIVERSITY OF SOUTHERN CALIFORNIA
}

\author{
STANFORD UNIVERSITY \\ UNIVERSITY OF UTAH \\ WASHINGTON STATE COLLEGE \\ UNIVERSITY OF WASHINGTON \\ AMERICAN MATHEMATICAL SOCIETY \\ CALIFORNIA RESEARCH CORPORATION \\ HUGHES AIRCRAFT COMPANY \\ THE RAMO-WOOLDRIDGE CORPORATION
}

Mathematical papers intended for publication in the Pacific Journal of Mathematics should be typewritten (double spaced), and the author should keep a complete copy. Manuscripts may be sent to any of the editors. All other communications to the editors should be addressed to the managing editor, E. G. Straus at the University of California, Los Angeles 24, California.

50 reprints per author of each article are furnished free of charge; additional copies may be obtained at cost in multiples of 50 .

The Pacific Journal, of Mathematics is published quarterly, in March, June, September, and December. The price per volume (4 numbers) is $\$ 12.00$; single issues, $\$ 3.50$. Back numbers are available. Special price to individual faculty members of supporting institutions and to individual members of the American Mathematical Society: $\$ 4.00$ per volume; single issues, $\$ 1.25$.

Subscriptions, orders for back numbers, and changes of address should be sent to Pacific Journal of Mathematics, 2120 Oxford Street, Berkeley 4, California.

Printed at Kokusai Bunken Insatsusha (International Academic Printing Co., Ltd.), No. 10, 1-chome, Fujimi-cho, Chiyoda-ku, Tokyo, Japan.

PUBLISHED BY PACIFIC JOURNAL OF MATHEMATICS, A NON-PROFIT CORPORATION

The Supporting Institutions listed above contribute to the cost of publication of this Journal, but they are not owners or publishers and have no responsibility for its content or policies. 


\section{Pacific Journal of Mathematics}

\section{Vol. 7, No. 2 \\ February, 1957}

William F. Donoghue, Jr., The lattice of invariant subspaces of a completely continuous quasi-nilpotent transformation ................... 1031

Michael (Mihály) Fekete and J. L. Walsh, Asymptotic behavior of restricted extremal polynomials and of their zeros.................... 1037

Shaul Foguel, Biorthogonal systems in Banach spaces ............... 1065

David Gale, A theorem on flows in networks ................... 1073

Ioan M. James, On spaces with a multiplication .................. 1083

Richard Vincent Kadison and Isadore Manual Singer, Three test problems in operator theory .................................... 1101

Maurice Kennedy, A convergence theorem for a certain class of Markoff processes........................................ 1107

G. Kurepa, On a new reciprocity, distribution and duality law ........ 1125

Richard Kenneth Lashof, Lie algebras of locally compact groups ........ 1145

Calvin T. Long, Note on normal numbers .................... 1163

M. Mikolás, On certain sums generating the Dedekind sums and their reciprocity laws ..................................... 1167

Barrett O'Neill, Induced homology homomorphisms for set-valued maps......................................... 1179

Mary Ellen Rudin, A topological characterization of sets of real numbers........................................... 1185

M. Schiffer, The Fredholm eigen values of plane domains 1187

F. A. Valentine, A three point convexity property .........

Alexander Doniphan Wallace, The center of a compact lattice is totally

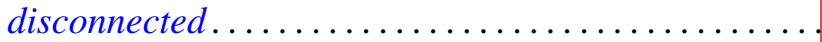

Alexander Doniphan Wallace, Two theorems on topological lattices.

G. T. Whyburn, Dimension and non-density preservation of mappings...

John Hunter Williamson, On the functional representation of certain algebraic systems ... 